\title{
Potential anticancer effect of prostratin through SIK3 inhibition
}

\author{
DALAL ALOTAIBI $^{1}$, SUNEETHA AMARA ${ }^{2}$, TERRANCE L. JOHNSON ${ }^{1}$ and VENKATASWARUP TIRIVEEDHI ${ }^{1,3}$ \\ ${ }^{1}$ Department of Biological Sciences, Tennessee State University, Nashville, TN 37209; ${ }^{2}$ Department of Medicine,
} St Thomas-Midtown, Nashville, TN 37203; ${ }^{3}$ Department of Cancer Biology, Vanderbilt University, Nashville, TN 37235 , USA

Received August 22, 2017; Accepted November 29, 2017

DOI: $10.3892 / \mathrm{ol} .2017 .7674$

\begin{abstract}
Prostratin, a phorbol ester natural plant compound, has been demonstrated to exert an anti-retroviral effect through activation of latent cluster of differentiation (CD)4+T lymphocytes and inhibition of viral entry into the cell through downregulation of chemokine receptor type 4 (CXCR4) expression. However, the potential effect of prostratin on cancer is yet to be defined. As CXCR4 is well known to induce cancer migration, it was hypothesized that prostratin induces an anti-cancer effect through inhibition of CXCR4 expression. The authors previously demonstrated that high stimulating conditions (sub-minimal IL-17, $0.1 \mathrm{ng} / \mathrm{ml}$, synergized with high salt, $\Delta 0.05 \mathrm{M} \mathrm{NaCl}$ ) promote breast cancer cell proliferation and CXCR4 expression through upregulation of salt-inducible kinase (SIK)-3. The present study demonstrated that prostratin selectively exerted increased cytotoxicity (IC50 of $7 \mu \mathrm{M}$ ) when breast cancer cells were cultured in high stimulating conditions, compared with regular basal culture conditions (IC50 of $35 \mu \mathrm{M}$ ). Furthermore, the cytotoxic potential of prostratin was increased seven-fold in the four breast cancer cell lines (MCF-7, MDA-MB-231, BT-20 and AU-565) compared with the non-malignant MCF10A breast epithelial cell line. This suggested that prostratin specifically targets cancer cells over normal cells. Mechanistic studies revealed that prostratin inhibited CXCR4 expression in breast cancer cells through downregulation of SIK3 expression. Overall, the data suggest that prostratin is a novel drug target for the pro-oncogenic factor SIK3. These studies could form a basis for further research to evaluate the anticancer effect of prostratin in a combinatorial chemotherapeutic regimen.
\end{abstract}

Correspondence to: Dr Venkataswarup Tiriveedhi, Department of Biological Sciences, Tennessee State University, 3500 John A Merritt Blvd, Nashville, TN 37209, USA

E-mail: vtirivee@tnstate.edu

Abbreviations: SIK3, salt inducible kinase-3; IL, interleukin; $\mathrm{NaCl}$, sodium chloride/salt; HIV, human immunodeficiency virus; CXCR4, C-X-C chemokine receptor type 4

Key words: breast cancer, chemokine, inflammation, interleukin-17, prostratin, salt inducible kinase-3

\section{Introduction}

Chronic inflammation is a well-established hallmark of cancer proliferation (1). The cellular stress caused by inflammation induces release of cytokine and chemokine factors which induce tumor progression and metastasis (2). Several agents have been suggested to induce chronic inflammation (3). Recent evidence from our lab have demonstrated that breast cancer cells cultured under high salt conditions $(\Delta 0.05 \mathrm{M}$ $\mathrm{NaCl}, 50 \%$ above basal culture conditions) were able to upregulate reactive nitrogen species (4,5). Importantly, sodium-MRI studies in breast cancer patients have demonstrated an increased sodium content, of up to $63 \%$ above the surrounding soft tissue, in the breast tumors $(6,7)$. These support a notion that high salt exerts an effector role on tumor progression, either working individually or synergistically to enhance an inflammatory tumor microenvironment. Phospho-proteomic based studies from our laboratory have demonstrated that high salt $(\Delta 0.05 \mathrm{M})$ synergized with sub-minimal stimulation of IL-17 $(0.1 \mathrm{ng} / \mathrm{ml})$ induced upregulation of SIK-3, salt inducible kinase-3, a serine-specific protein kinase in breast cancer cells (8). Mechanistic studies have demonstrated that SIK3 played a crucial role in induction of G1/S-phase release of cell cycle, along with enhanced expression of metastasis specific chemokine CXCR4 in breast cancer cells. Histone deacetylase (HDAC4) is a well-documented downstream target of SIK3 (9). The phosophorylation of HDAC4 is known to induce cell proliferation and malignancy. SIK3 induced phosphorylation of HDAC4. Further, equimolar treatment with mannitol or sucrose did not exert similar pro-cancer effect, thus strongly suggesting that high sodium chloride specifically induces a pro-cancer effect (8).

Natural plant product prostratin (12-deoxyphorbol 13 -acetate) is widely studied for it's activation of latent T-cells infected with HIV. Prostratin is identified as a pharmacologically active ingredient in the Samoan medicinal plant Homolanthus nutans (10). Molecular studies have demonstrated that prostratin exerts its anti-HIV effect through activation of protein kinase C (11). Importantly, prostratin is shown to induce downregulation of chemokine receptor CXCR4 in CD4+T lymphocytes there by preventing the entry of HIV-1 virus into lymphocytes (12).

The C-X-C chemokine receptor type 4 (CXCR4), belongs to the superfamily of the heterotrimeric $\mathrm{G}$ protein-coupled receptors and is expressed on the cell surface of various types of metastatic breast cancer cells. It is important to note that the 
role of CXCR4 in cell proliferation and metastasis was obtained from the elegant studies performed by Dewan et al (13), when less invasive MCF-7 breast cancer lines with low expression of CXCR4 formed much smaller tumor mass the SCID-mice compared to those larger tumor size the highly invasive MDA-MB-231 cell line with high expression of CXCR4. This is further corroborated with the studies by Lapteva et al (14), where in, siRNA based knock-down of CXCR4 in breast cancer cells tumors cells decrease the tumor growth and size in the murine breast cancer models. Taken together, as prostratin has shown to mediate CXCR4 downregulation in HIV infected cells and CXCR4 is important for cancer proliferation, in our current communication, we studied the anti-cancer effect of prostratin through modulation of CXCR4 expression on cancer cells.

\section{Materials and methods}

Cell cultures and plasmids. Five breast tissue related cell lines were used in our studies, of these, four breast cancer cells (MCF7, MDA-MB-231, BT20, AU565) and one normalized breast epithelial cell line (MCF10A) were utilized and obtained from the American Type Culture Collection (ATCC, Manassas, VA, USA). The cells were cultured in cell basal essential media (RPMI-1640 media; Thermo Fisher Scientific, Inc., Waltham, MA, USA) along with the media supplements such as fetal bovine serum, penicillin/streptomycin, fungizone, HEPES and glutamine, as recommended by the manufacturer and as previously described $(4,15)$. Cell lines were frozen in liquid vapor nitrogen at $-130^{\circ} \mathrm{C}$ until use. Upon thawing, cells were maintained in $5 \% \mathrm{CO}_{2}$ incubator in sterile essential media at $37^{\circ} \mathrm{C}$. For salt and interleukin-17 treatment conditions, cell culture media was supplemented with $0.05 \mathrm{M} \mathrm{NaCl}$ (Sigma-Aldrich; Merck KGaA, Darmstadt, Germany) and $0.1 \mathrm{ng} / \mathrm{ml}$ IL-17 (Thermo Fisher Scientific, Inc.). We have previously performed a dose-response for salt (0-0.1 $\mathrm{M} \mathrm{NaCl})$ and IL-17 (0-1,000 ng/ $\mathrm{ml}$ ) and found-out that $0.05 \mathrm{M} \mathrm{NaCl}$ provided highest cell proliferation $(4,5)$ and $0.1 \mathrm{ng} / \mathrm{ml}$ of IL-17 induced sub-effective inflammatory cytokine response $(4,16)$. All chemicals unless mentioned were obtained either from Sigma-Aldrich; Merck KGaA (Darmstadt, Germany) or Thermo Fisher Scientific, Inc. For siRNA knock down of SIK3 we have used the following two siRNA sequences: SIK3-siRNA-1: 5'-GUGCAGAGUGUU GGAGUCC-3'; scramble SIK3-siRNA-1: 5'-UGGAGGCGA GUCAGUUUGC-3'.

Western blot/immunoprecipitation. Total proteins were extracted from cells with lysis buffer for western blot analysis as previously described $(17,18)$. Total proteins were separated on a $4-12 \%$ sodium dodecyl sulfate-polyacrylamide gradient gel and transferred onto a nitrocellulose membrane. The membranes were blocked overnight at $4^{\circ} \mathrm{C}$ in Tris-buffered saline with $0.05 \%$ Tween 20 (5\% nonfat milk in 10 mM Tris-HCl-100 mM NaCl-0. $1 \%$ Tween 20, pH 7.4). The membranes were incubated first with Abs specific for total and phosphorylated forms at room temperature with primary Abs diluted 1 in 1,000 in blocking buffer for $2 \mathrm{~h}$, and then with a horseradish peroxide-conjugated secondary IgG mAb diluted 1 in 5,000 for $1 \mathrm{~h}$. All primary and secondary Abs were obtained from Santa Cruz Biotechnology, Inc., (Dallas, TX, USA). All primary and secondary antibodies for western blot and immunoprecipitation were obtained from either Abcam (Cambridge, MA, USA) or Santa Cruz Biotechnology, Inc. The following specific primary antibodies to SIK3 (ab211424; Abcam) GADPH (sc-47724), Actin (sc-8432), HDAC4 (sc-46672; all from Santa Cruz Biotechnology, Inc.), pHDAC4-S632 (ab39408; Abcam). The membrane was developed using the chemiluminescence kit (EMD Millipore, Billerica, MA, USA) and analyzed on using Universal Hood II by Bio-Rad Laboratories, Inc., (Hercules, CA, USA). Morphometric analysis was done using the software provided by the company.

For SIK3 immunoprecipitation, the cultured cells were washed with cold PBS, and lysed for $30 \mathrm{~min}$ on ice with $0.5 \mathrm{ml}$ of lysis buffer as previously mentioned $(16,19)$. To the lysis buffer $0.5 \mathrm{ml}$ of dilution buffer was added and centrifuged at $17,000 \mathrm{x} \mathrm{g}$ for $30 \mathrm{~min}$. The supernatant was transferred and $1 \mu \mathrm{g}$ normal chicken IgY (ab97135; Abcam) or chicken anti-SIK3 were added. After overnight incubation at $4^{\circ} \mathrm{C}, 30 \mu \mathrm{l}$ carbolink beads (Pierce, Thermo Fisher Scientific, Inc.) were added to lysates and incubated for $2 \mathrm{~h}$ for chicken antibody immunoprecipitation as per manufacturer's protocol. Beads were washed with $700 \mu \mathrm{l}$ of wash ice cold buffer four times, $3 \mathrm{~min}$ each time followed by centrifugation at $1,800 \mathrm{x} \mathrm{g}$ for 3 min at $4^{\circ} \mathrm{C}$. Beads were then washed with cold PBS and bound proteins were eluted by boiling with $30 \mu \mathrm{l}$ of $2 \mathrm{X}$ SDS buffer for $10 \mathrm{~min}$. Proteins were subjected to SDS-PAGE (4-12\% gel) and immunoblotting. Phosphorylation of SIK3 were detected with a mouse monoclonal phospho-serine antibody (sc-81514; Santa Cruz Biotechnology, Inc.).

Reverse transcription-quantitative polymerase chain reaction $(R T-q P C R)$. Expression profiles of genes at mRNA level in the breast cancer cell lines were analyzed using the TaqMan FAM-labeled RT-PCR primers for SIK3 (Hs00228549_m1), GADPH (Hs402869), and Actin (Hs4333762T), obtained from Applied Biosystems; Thermo Fisher Scientific, Inc. as per the manufacturer's recommendation. Briefly, total RNA was extracted from $10^{6}$ cells using TRIzol reagent (Sigma-Aldrich; Merck KGaA) and analyzed as mentioned previously (20-22). RNA samples were quantified by absorbance at $260 \mathrm{~nm}$. The RNA was reverse-transcribed and RT-PCR (real time PCR) was performed in a final reaction volume of $20 \mu \mathrm{l}$ using CFX96 (Bio-Rad Laboratories, Inc.). Each sample was analyzed in triplicate. Cycling conditions consisted of an initial denaturation of $95^{\circ} \mathrm{C}$ for $15 \mathrm{~min}$, followed by 40 cycles of $95^{\circ} \mathrm{C}$ for $30 \mathrm{sec}$, followed by $61^{\circ} \mathrm{C}$ for $1 \mathrm{~min}$.

Cell proliferation assay. Cell viability was measured by trypan blue dye exclusion (Sigma-Aldrich; Merck KGaA) and MTT assay (Thermo Fisher Scientific, Inc.) as previously described (4). Briefly, the viability of breast cancer cells was assessed by measuring mitochondrial activity using MTT (4,5-dimethylthiazol-2-yl) 2,5-diphenyltetrazolium bromide) assay. For various treatment conditions the cancer cells were plated in 96 well plate for 48-72 h, the cells were incubated with $5 \mathrm{mg} / \mathrm{ml} \mathrm{MTT}$ in PBS for $2 \mathrm{~h}$, latter lysed with manufacturer provided reagents. Detection at $570 \mathrm{~nm}$ was performed using EMax Plus spectrophotometer and data analysis was carried out using software provided by the manufacturer (Molecular Devices, LLC, Sunnyvale, CA, USA). Viability was calculated 


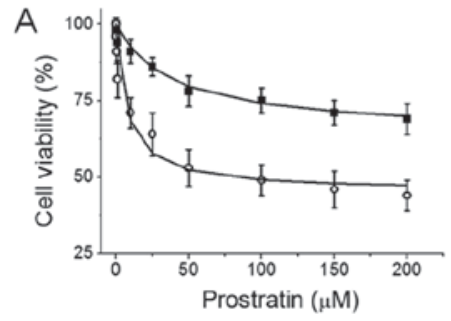

\begin{tabular}{|c|c|c|c|c|}
\hline & \multicolumn{2}{|c|}{ Unstimulated } & \multicolumn{2}{|c|}{ Stimulated } \\
\hline & IC50 ( $\mu \mathrm{g})$ & $\mathrm{C}_{\max }(\%)$ & $\mathrm{ICSO}(\mu \mathrm{g})$ & $\mathrm{C}_{\max }(\%)$ \\
\hline MCF-7 & $37.4 \pm 8.7$ & $35.4 \pm 5.3$ & $7.3 \pm 2.9^{*}$ & $54.6 \pm 4.6^{*}$ \\
\hline MDA-MB-231 & $31.9 \pm 6.3$ & $42.7 \pm 6.1$ & $6.6 \pm 2.7^{*}$ & $63.4 \pm 5.2^{*}$ \\
\hline BT-20 & $28.1 \pm 10.3$ & $45.9 \pm 4.8$ & $6.3 \pm 3.2^{*}$ & $69.1 \pm 4.5^{*}$ \\
\hline AU565 & $44.6 \pm 9.2$ & $35.4 \pm 5.3$ & $7.7 \pm 3.8^{*}$ & $48.9 \pm 6.2^{*}$ \\
\hline MCF10A & $73.7 \pm 13.9$ & $21.4 \pm 3.2$ & $49.2 \pm 11.8^{*}$ & $27.4 \pm 6.7$ \\
\hline
\end{tabular}

Figure 1. Prostratin induces cytotoxicity in breast cancer cells. (A) Dose titration of prostratin (0 to $200 \mu \mathrm{M})$ on MCF-7 cells; (filled squares) represent cells cultured under basal conditions; (open circles) represent cells cultured under high salt stimulating conditions ( $\Delta 0.05 \mathrm{M} \mathrm{NaCl}+0.1 \mathrm{ng} / \mathrm{ml} \mathrm{IL}-17)$. (B) Dose titration was performed on the five breast cell lines, of which four (MCF-7, MDA-MB-231, BT-20, AU-565) are breast cancer lines and MCF-10 A is non-malignant breast epithelial cell line. Data were curve fit using the equation provided in methods section. IC50 and $\mathrm{C}_{\max }$ were obtained are presented above. One-way ANOVA analysis was performed for statistical analysis to determine the statistical significance of IC50 and $\mathrm{C}_{\text {max }}$ for comparison between unstimulated and stimulated groups; significance taken at $\mathrm{P}<0.05$, represented by astericks $\left(^{*}\right)$.

as percentage compared to untreated cells. Drug dose-reponse parameters were obtained using the following equation:

$$
y=100-\left[A^{*} x /(K+x)\right]
$$

where, $y$ is cell viability $(\%) ; x$ is prostratin concentration $(\mu \mathrm{M})$; $A$ is defined as the maximum cell viability following highest drug treatment, this data will be reported as maximal prostratin cytoxicity $\left(\mathrm{C}_{\max }\right)$, as represented as $(100-\mathrm{A}) ; K$ is defined as the concentration of prostratin at which there is $50 \%$ of $\mathrm{C}_{\max }$ loss of cell viability and is reported as IC50. While Cmax and IC50 are obtained by best curve fit (with R-square value $>0.95$ ), the highest drug treatment within the limitation of our experimental data collection is shown at approximately three fold concentration above IC50. The best curve fit was analyzed using Microcal Origin v7.0 (Microcal Software, Westborough, MA, USA).

HDAC4 assay kit. The HDAC4 activity analysis (Epigentek, Farmingdale, NY, USA) was performed on the nuclear fragments of the cell lysates under various assay conditions as per manufacturer's instructions. Calorimetry detection at $450 \mathrm{~nm}$ was performed using EMax Plus spectrophotometer and data analysis was carried out using software provided by the manufacturer (Molecular Devices, LLC). The data analysis was performed based on a standard curve obtained using the positive controls provided by the manufacturer as previously described.

CXCR4 membrane expression assay. CXCR4 expression was analyzed by flow cytometry as previously described (23). Briefly, the CXCR4 protein was labeled by mouse anti-CXCR4 primary antibody (Santa Cruz Biotechnology, Inc.) in 1:20 dilution to a $200 \mu \mathrm{l}$ final volume of cells $\left(1 \times 10^{5}\right.$ cells $\left./ \mathrm{ml}\right)$. Antibodies used for flow cytometry included anti-mouse-FITC (BD Biosciences, San Jose, CA, USA), and the samples were latter analyzed using a FACS Calibur/LSRII flow cytometer (BD Biosciences). Data were analyzed using BD FACSDiva software. Gates were set according to isotype controls.

Statistical analysis. All data were presented as mean values \pm SEM from four independent experiments. Student $\mathrm{t}$-test performed for statistical analysis. $\mathrm{P}<0.05$ was considered to indicate a statistically significant difference. All data analysis were performed using Origin 6 software (Origin
Labs, Northampton, MA, USA) or SPSS software v21 (IBM Corp., Armonk, NY, USA).

\section{Results}

Enhanced cytotoxic effect of prostratin on breast cancer cells in the presence of high salt environment. To study the effect on prostratin on breast cancer cells in the presence on high salt environment, we first determined the drug's cytotoxicity. We have previously demonstrated that high salt $(\Delta 0.05 \mathrm{M}$ $\mathrm{NaCl})$ synergized with subminimal IL-17 (0.1 pg/ml) (16) to induce a $24 \%$ enhanced cell proliferation $(4,5,8,22)$, which will be referred to as stimulating conditions in our current communication. Importantly, Na-MRI based human studies have revealed that breast tumors accumulate high salt for yet unknown reasons $(6,7)$. As shown in Fig. 1A, prostratin induced inhibitory effect on cell proliferation in MCF-7 breast cancer cells, however, this cytotoxic effect was higher when cells were culture in high salt culture environment. Further data analysis has revealed that (Fig. 1B) the IC50 of prostratin on MCF-7 cells under basal culture conditions was $37.4 \pm 8.7 \mu \mathrm{g}$, while under high salt stimulating conditions the IC50 was determined to be $7.3 \pm 2.9 \mu \mathrm{g}$. This suggests that natural plant product prostratin has more efficient anti-tumor activity under tumor microenvironment conditions similar to real human cancer patients. We have further verified this cytotoxic effect on other breast related cell lines. In our current study we have used four breast cancer cell lines namely, MCF-7 (ER/PR double positive), MDA-MB-231 (triple negative), BT-20 (Triple negative), AU-565 (Her2 positive); and one non-malignant breast epithelial cell line, MCF-10A. As shown in Fig. 1B, prostratin has upto seven fold higher cytotoxicity (as determined by low IC50) on breast cancer cells over non-malignant cells. Of the four breast cancer cell lines, although statistically insignificant, prostratin seemed to exert higher effect (as determined by $\mathrm{C}_{\max }$ ) on the two highly invasive (metastatic) cell lines, MDA-MD-231 and BT-20 (Fig. 1B). These data strongly suggest that prostratin can selectively exert it's effect on breast tumor with minimal effect on normal breast epithelium.

Prostratin inhibits SIK3 expression and phosphorylation. As we have previously demonstrated that SIK3 plays a critical role in mediating high salt induced cancer cell proliferation (8). In our current study, we studied the potential cytotoxic effect of 
A

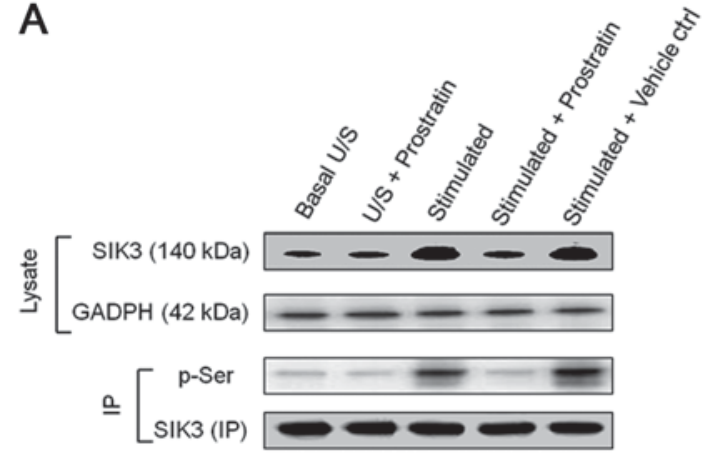

B

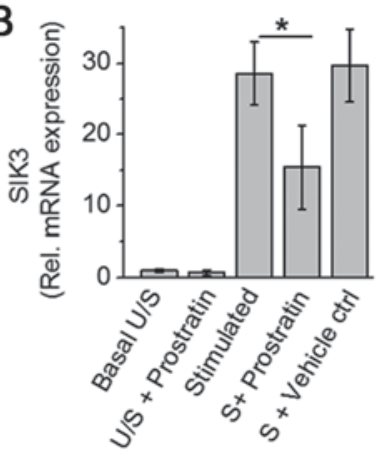

C

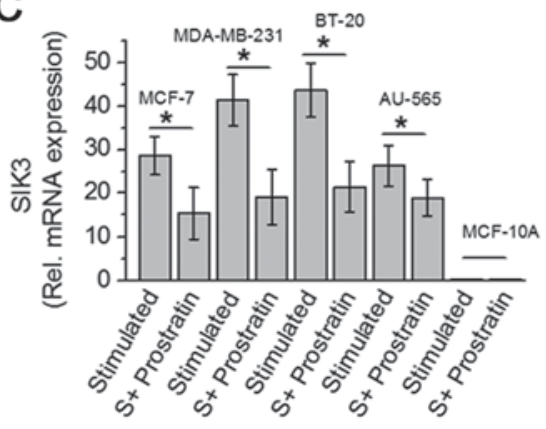

Figure 2. Prostratin inhibits SIK3 expression and phosphorylation in breast cancer cells. (A) Westernblot analysis of SIK3 expression in the total cell lysate of MCF7 cells. Immunoprecipitate of SIK3 was probed with phosphor-serine antibody; U/S, represents unstimulated condition, i.e., cells cultured under basal conditions; S, represent cells cultured under high salt stimulating conditions $(\Delta 0.05 \mathrm{M} \mathrm{NaCl}+0.1 \mathrm{ng} / \mathrm{ml} \mathrm{IL}-17)$; Prostratin treatment concentration was $50 \mu \mathrm{M}$; equi-volume DMSO was used as vehicle control. (B) SIK3 mRNA transcript expression analysis in MCF-7 cells performed by quantitative RT-PCR. (C) SIK3 mRNA transcript expression analysis in five breast cell lines mentioned above following high salt stimulating conditions with and without $50 \mu \mathrm{M}$ prostratin. Student-t-test performed for statistical analysis; significance taken at $\mathrm{P}<0.05$, represented by astericks $\left({ }^{*}\right)$.

A

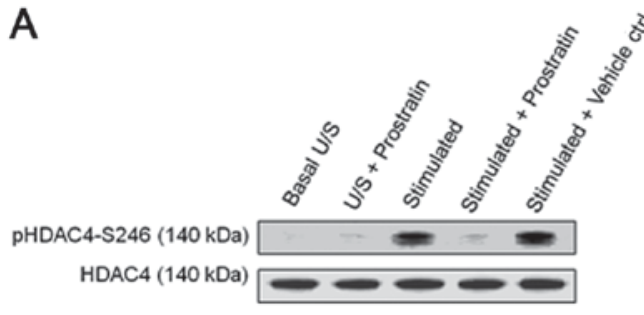

B

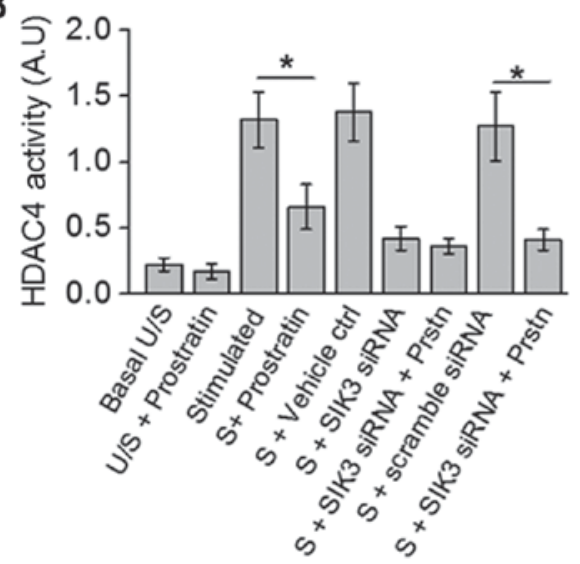

C

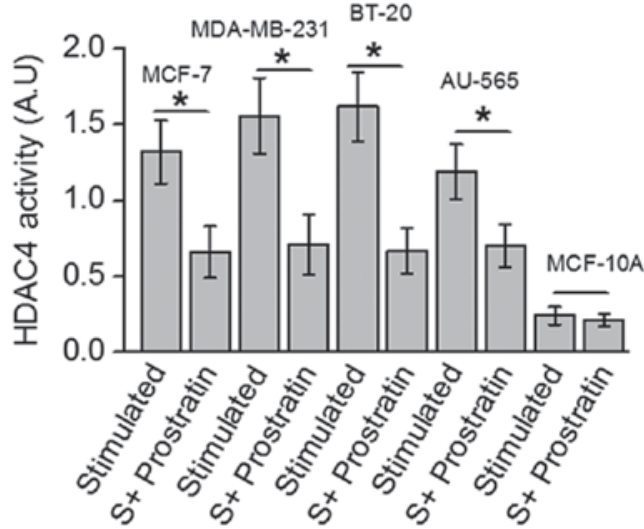

Figure 3. Prostratin inhibits HDAC4 phosphorylation in breast cancer cells. (A) Westernblot analysis of HDAC4 phosphorylation in the total cell lysate of MCF7 cells. (B) ELISA-based HDAC4 activity analysis performed in MCF-7 cells under various conditions mentioned above. (C) ELISA-based HDAC4 activity analysis performed in five breast cell lines mentioned above following high salt stimulating conditions with and without $50 \mu \mathrm{M}$ prostratin. Student-t-test performed for statistical analysis; significance taken at $\mathrm{P}<0.05$, represented by astericks $\left(^{*}\right)$.

prostratin through inhibition of SIK3. As shown in Fig. 2, prostratin induced a $46 \%$ inhibition in the SIK3 mRNA expression following stimulation with high salt on MCF-7 cells. Prostratin was able to exert this SIK3 inhibition on all four breast cancer cells (MCF-7, MDA-MB-231, BT-20 and AU-565). Similar to evidence from cytotoxicity studies, prostratin exerted slightly greater inhibition (54\% vs. $46 \%$ inhibition, $\mathrm{P}>0.05$, statistically non-significant) of SIK3 expression in highly invasive MDA-MB-231 and BT-20, over less invasive MCF-7 and AU-565 breast cancer cell lines. Furthermore, along with reduced SIK3 expression prostratin also inhibited the phosphorylation of SIK3 suggesting an inhibition of SIK3 mediated downstream signaling.
Prostratin inhibits activation of HDAC4, a SIK3 downstream element. Previous studies in our laboratory have demonstrated that high salt stimulating conditions induce SIK3 mediated phosphorylation of HDAC4, and siRNA mediated knock-down of SIK3 completely abrogated HDAC4 phosphorylation (8). Towards this, as prostratin inhibited SIK3, we studied the drug effect on HDAC4 phosphorylation, a SIK3 downstream element. As shown in Fig. 3A, prostratin inhibited the phosphorylation of HDAC4. Further, ELISA based biochemical analysis of HDAC4 activity (Fig. 3B) demonstrated that prostratin treated cell lysate obtained following treatment with high salt stimulation induced a $50 \%$ inhibition of HDAC4 specific substrate conversion. 

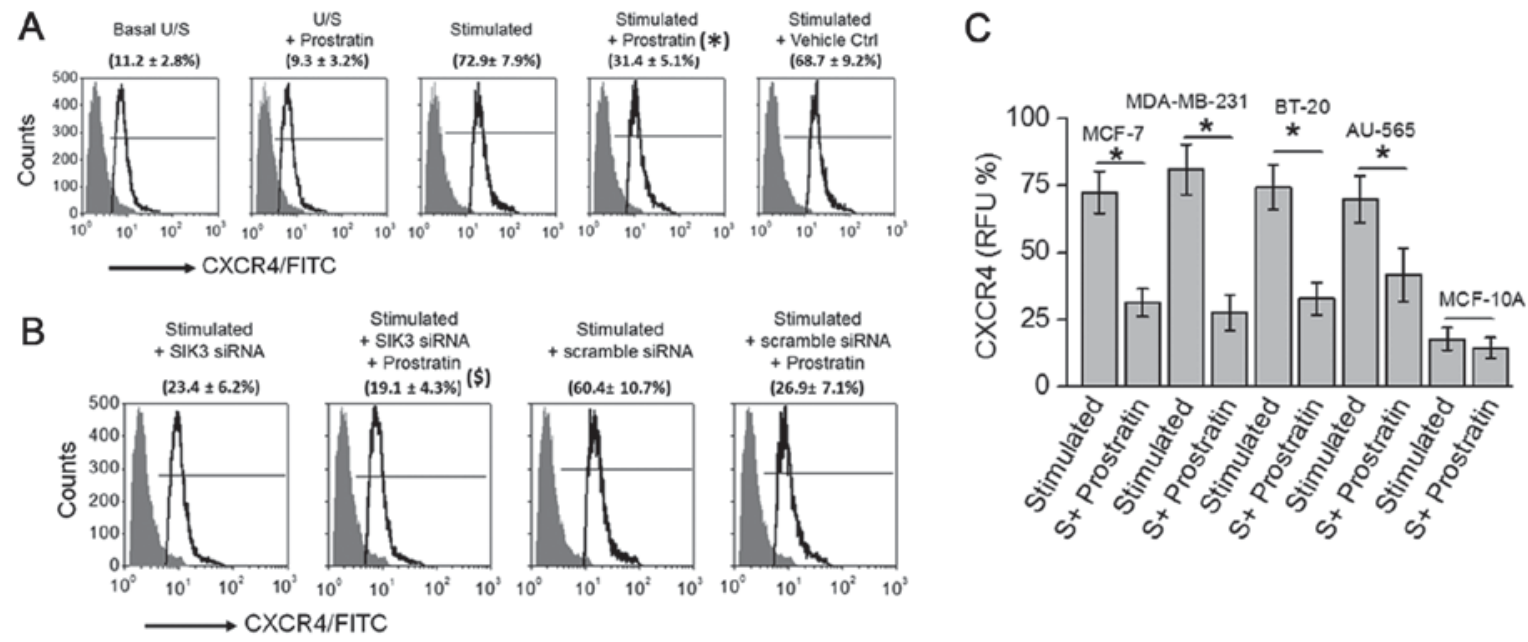

Figure 4. Prostratin inhibits CXCR4 expression in breast cancer cells. (A) Flow cytometry based analysis for expression of CXCR4 in MCF7 cells. (B) CXCR4 expression following treatment with SIK3 siRNA and scramble siRNA. (\$) represents statistical significance made by comparison between (stimulated+scramble siRNA) vs. (stimulated+scramble siRNA+50 $\mu \mathrm{M}$ prostratin) samples. (C) CXCR4 expression analysis performed in five breast cell lines mentioned above following high salt stimulating conditions with and without $50 \mu \mathrm{M}$ prostratin. Student-t-test performed for statistical analysis; significance taken at $\mathrm{P}<0.05$. (") represents statistical significance made by comparison between stimulated and stimulated+prostratin samples.

Taken together, these data indicate that prostratin exerts its anti-tumor effect through inhibition of SIK3/HDAC4 mediated cell proliferation.

Prostratin downregulates CXCR4 in SIK3 dependent manner. As our cytotoxicity and SIK3 functional mechanistic data suggested that prostratin exerted a slightly higher (but statistically insignificant) inhibitory effect on highly invasive MDA-MB-231 and BT-20 cancer cell lines, we tested if prostratin could exerted anti-cancer effect through inhibition of metastasis. CXCR4 is a well-established metastatic factor which is known to promote cancer cell invasion and spreading to other organs in patients (24). Importantly, prostratin has previously been demonstrated to inhibit CXCR4 expression in T-lymphocytes. Therefore, in the present study we tested if prostratin inhibited CXCR4 expression in breast cancer cells. As shown in Fig. 4A, prostratin treatment inhibited the CXCR4 expression (from $72.9 \pm 7.9 \%$ to $31.4 \pm 5.1 \%$; $\mathrm{P}<0.05$ ) following high salt stimulation. Prostratin exerted no effect following siRNA mediated SIK3 knock down, suggesting prostratin inhibits SIK3 induced CXCR4 metastatic factor expression. Further, prostratin was able to inhibit CXCR4 in all four breast cancer cell lines (Fig. 4C) with no effect on non-malignant breast epithelial cell line. These data suggest that prostratin is exerts strong anti-cancer effect through potential inhibition of metastasis.

\section{Discussion}

The natural product prostratin is extensively researched in the context of therapy against HIV infection and activation of CD4+T lymphocytes. However, there is limited evidence to determine the effect of prostratin on cancer cells. Various ligand-drug interaction studies have suggested that prostratin exerts its anti-viral effect through the diacylglycerol (DAG) binding domain of protein kinase $\mathrm{C}$ (PKC), leading to PKC enzymatic activation (11). This logic behind ligand mechanistics of prostratin, a phorbol ester, seems to have to been inspired from the understanding of another structurally related phorbol ester, phorbol 12-myristate 13-acetate (PHA), which also stimulates PKC through allosteric activation by DAG. However, inspite of structural similarity, while PHA is known to promote tumor growth, prostratin is not considered to promote tumor growth. Szallasi et al (25) have demonstrated that prostratin was able to inhibit tumor growth in CD1 murine cutaneous tumor models. However, to-date limited literature on the exact cytotoxicity doses of prostratin on cancer cell lines. In our current study we report that the IC50 of prostratin on four different breast cancer cells under basal culture conditions was around $35 \mu \mathrm{M}$ (Fig. 1) however, upon stimulating culture conditions, mimicking the real tumor microenvironment, the IC50 was around $7 \mu \mathrm{M}$ (Fig. 1), thus suggesting that prostratin might have an anticancer effect under in vivo solid tumor environment in animal models and humans. Further, it is important to note that the IC50 of prostratin on non-malignant breast cells (MCF-10A) under basal culture conditions (which is close to basal physiological conditions within human body) is $73 \mu \mathrm{M}$ (Fig. 1). This data strongly suggests that prostratin has a 10 fold higher therapeutic index and therefore could specifically target malignant cells while being safe on normal cells. This data could set a stage for future preclinical studies in murine tumor models to exactly decipher the anticancer effect and therapeutic dose of prostratin.

To date, extensive research with prostratin as an anti-viral drug has demonstrated that the drug exerts its effect through activation of PKC (11). However, using fibroblast cell lines stably transfected with H-Ras and K-Ras, Wang et al (26) have demonstrated yet another mechanism for prostratin, wherein the researchers have demonstrated that prostratin inhibits K-Ras/calmodulin interaction and there by inhibiting the oncogenic potential of their transfected cell lines. This evidence suggests, in addition to PKC activation, prostratin could exert its anti-cancer effect through other signaling pathways. In the present study as prostratin demonstrated higher cytotoxicity when cells were cultured under stimulating 
conditions (sub-minimal IL-17, $0.1 \mathrm{ng} / \mathrm{ml}$, synergized high salt, $\Delta 0.05 \mathrm{M} \mathrm{NaCl}$, culture conditions) commonly found in solid tumors. This data suggest that prostratin might act through salt-specific signaling mechanism. Importantly, we have recently demonstrated that salt-inducible kinase, SIK3, is specifically upregulated and mediated proliferative signaling following high salt stimulating conditions. Therefore, we tested for the potential effect of prostratin on SIK3 expression. Previous studies from our laboratory have demonstrated that (8), while IL-17 induced both mTORC1 and mTORC2 pathways, only mTORC2 is a direct upstream signaling molecule for SIK3. High salt treatment could directly induce SIK3 phosphorylation, which is further enhanced following co-treatment with subminimal-IL-17. These studies clearly point out that SIK3 is a direct downstream factor for high salt synergized inflammatory stress. In our current study as we found prostratin could down-regulate the activation of SIK3, we wanted to study under treatment conditions wherein we had highest stimulation of SIK3 and then quantitatively analyze for the prostratin inhibition of SIK3. Therefore, as the focus of the current study is SIK3, to have highest stimulation of SIK3 in our current work, we have used high salt and sub-minimal IL-17 co-treatment conditions. Our current studies demonstrate that prostratin inhibits SIK3 expression and phosphorylation (Fig. 2). Also, the phosphorylation and activity of histone deacetylase (HDAC4), a direct downstream molecule of SIK3 (9), was inhibited by prostratin (Fig. 3). Taken together, these data strongly suggest that prostratin inhibits SIK3 mediated pro-cancer signaling. However, more elaborate studies in the preclinical SIK3 knock-out animal models and detailed cell cycle studies would be warranted to delineate the effect of prostratin on SIK3 mediated signaling in solid tumors.

Prostratin had been shown to elicit anti-HIV effect by inducing down-regulation of CD4, C-X-C chemokine receptor type 4 (CXCR4), and thereby protecting $\mathrm{CD} 4+\mathrm{T}$ cells from HIV-1 entry (12). In acutely infected cells, prostratin is thought to enhance cellular protection possibly due to cytostatic effects (27). In the context of cancer CXCR4 is well-known to induce cancer cell migration and metastasis (24). Elevated membrane expression of CXCR4 is observed in several cancers. Further CXCR4 is positively correlated with cancer progression and considered a poor prognostic biomarker (28). Several factors contribute to the upregulation of CXCR4 in malignant cells. Previous studies in our laboratory have demonstrated that, under high salt stimulating conditions, enhanced SIK3 signaling through MMP-9 pathway mediates upregulation of CXCR4 membrane expression (8). In our current studies we demonstrate that prostratin inhibits CXCR4 expression in breast cancer cell lines (Fig. 4). Furthermore, siRNA mediated knockdown of SIK3 under high salt stimulating culture conditions did not induce expression of CXCR4, thus suggesting that, atleast that under our experimental conditions, prostratin mediated CXCR4 inhibition is mediated by the direct drug induced downregulation of SIK3.

In conclusion, we demonstrated a novel mechanism of action for prostratin. SIK3 inhibition could be a novel area of drug-target anti-cancer studies. In addition to PKC activation, prostratin could exert its anti-cancer effect through inhibition of SIK3. This data could provide a mechanistic basis for further research to study the potential application of prostratin as an add-on drug to the anti-cancer chemotherapeutic regimen.

\section{Acknowledgements}

This study was supported by NIH grant no. 2U54-CA163066-06 (cancer partnership grant). The authors would like to thank Department of Biological Sciences, Tennessee State University for their financial support.

\section{References}

1. Crusz SM and Balkwill FR: Inflammation and cancer: Advances and new agents. Nat Rev Clin Oncol 12: 584-596, 2015.

2. Hanahan D and Weinberg RA: Hallmarks of cancer: The next generation. Cell 144: 646-674, 2011.

3. Landskron G, De la Fuente M, Thuwajit P, Thuwajit C and Hermoso MA: Chronic inflammation and cytokines in the tumor microenvironment. J Immunol Res 2014: 149185, 2014.

4. Amara S, Ivy MT, Myles EL and Tiriveedhi V: Sodium channel $\gamma \mathrm{ENaC}$ mediates IL-17 synergized high salt induced inflammatory stress in breast cancer cells. Cell Immunol 302: 1-10, 2016.

5. Amara S, Zheng $\mathrm{M}$ and Tiriveedhi V: Oleanolic acid inhibits high salt-induced exaggeration of warburg-like metabolism in breast cancer cells. Cell Biochem Biophys 74: 427-434, 2016.

6. Ouwerkerk R, Jacobs MA, Macura KJ, Wolff AC, Stearns V Mezban SD, Khouri NF, Bluemke DA and Bottomley PA: Elevated tissue sodium concentration in malignant breast lesions detected with non-invasive 23Na MRI. Breast Cancer Res Treat 106: 151-160, 2007.

7. Zaric O, Pinker K, Zbyn S, Strasser B, Robinson S, Minarikova L, Gruber S, Farr A, Singer C, Helbich TH, et al: Quantitative sodium MR Imaging at $7 \mathrm{~T}$ : Initial results and comparison with diffusion-weighted imaging in patients with breast tumors. Radiology 280: 39-48, 2016.

8. Amara S, Majors C, Roy B, Hill S, Rose KL, Myles EL and Tiriveedhi V: Critical role of SIK3 in mediating high salt and IL-17 synergy leading to breast cancer cell proliferation. PLoS One 12: e0180097, 2017.

9. Walkinshaw DR, Weist R, Kim GW, You L, Xiao L, Nie J, Li CS Zhao S, Xu M and Yang XJ: The tumor suppressor kinase LKB1 activates the downstream kinases SIK2 and SIK3 to stimulate nuclear export of class IIa histone deacetylases. J Biol Chem 288 : 9345-9362, 2013.

10. Gustafson KR, Cardellina JH II, McMahon JB, Gulakowski RJ, Ishitoya J, Szallasi Z, Lewin NE, Blumberg PM, Weislow OS, Beutler JA, et al: A nonpromoting phorbol from the samoan medicinal plant homalanthus nutans inhibits cell killing by HIV-1. J Med Chem 35: 1978-1986, 1992.

11. Shen X, Xiong GL, Jing Y, Xiao H, Cui Y, Zhang YF, Shan YJ, Xing S, Yang M, Liu XL, et al: The protein kinase $\mathrm{C}$ agonist prostratin induces differentiation of human myeloid leukemia cells and enhances cellular differentiation by chemotherapeutic agents. Cancer Lett 356: 686-696, 2015.

12. Hezareh M, Moukil MA, Szanto I, Pondarzewski M, Mouche S, Cherix N, Brown SJ, Carpentier JL and Foti M: Mechanisms of HIV receptor and co-receptor down-regulation by prostratin: Role of conventional and novel PKC isoforms. Antivir Chem Chemother 15: 207-222, 2004.

13. Dewan MZ, Ahmed S, Iwasaki Y, Ohba K, Toi M and Yamamoto N: Stromal cell-derived factor-1 and CXCR4 receptor interaction in tumor growth and metastasis of breast cancer. Biomed Pharmacother 60: 273-276, 2006.

14. Lapteva N, Yang AG, Sanders DE, Strube RW and Chen SY: CXCR4 knockdown by small interfering RNA abrogates breast tumor growth in vivo. Cancer Gene Ther 12: 84-89, 2005.

15. Tiriveedhi V, Gelman AE and Mohanakumar T: HIF-1 $\alpha$ signaling by airway epithelial cell K- $\alpha 1$-tubulin: Role in fibrosis and chronic rejection of human lung allografts. Cell Immunol 273: 59-66, 2012.

16. Amara S, Lopez K, Banan B, Brown SK, Whalen M, Myles E, Ivy MT, Johnson T, Schey KL and Tiriveedhi V: Synergistic effect of pro-inflammatory TNF $\alpha$ and IL-17 in periostin mediated collagen deposition: Potential role in liver fibrosis. Mol Immunol 64: 26-35, 2015. 
17. Tiriveedhi V, Tucker N, Herndon J, Li L, Sturmoski M, Ellis M Ma C, Naughton M, Lockhart AC, Gao F, et al: Safety and preliminary evidence of biologic efficacy of a mammaglobin-a DNA vaccine in patients with stable metastatic breast cancer. Clin Cancer Res 20: 5964-5975, 2014.

18. Tiriveedhi V, Angaswamy N, Brand D, Weber J, Gelman AG, Hachem R, Trulock EP, Meyers B,Patterson G and Mohanakumar T: A shift in the collagen $\mathrm{V}$ antigenic epitope leads to $\mathrm{T}$ helper phenotype switch and immune response to self-antigen leading to chronic lung allograft rejection. Clin Exp Immunol 167: 158-168, 2012.

19. Sarma NJ, Tiriveedhi V, Crippin JS, Chapman WC and Mohanakumar T: Hepatitis C virus-induced changes in microRNA 107 (miRNA-107) and miRNA-449a modulate CCL2 by targeting the interleukin- 6 receptor complex in hepatitis. J Virol 88: 3733-3743, 2014.

20. Tiriveedhi V, Takenaka M, Ramachandran S, Gelman AE, Subramanian V, Patterson GA and Mohanakumar T: T regulatory cells play a significant role in modulating MHC class I antibody-induced obliterative airway disease. Am J Transplant 12: 2663-2674, 2012.

21. Tiriveedhi V, Takenaka M, Sarma NJ, Gelman AG and MohanakumarT: Anti-major histocompatibility complex-induced obliterative airway disease: Selective role for CD4 and CD8 T cells in inducing immune responses to self-antigens. J Heart Lung Transplant 32: 714-722, 2013.

22. Amara S, Alotaibi D and Tiriveedhi V: NFAT5/STAT3 interaction mediates synergism of high salt with IL-17 towards induction of VEGF-A expression in breast cancer cells. Oncol Lett 12: 933-943, 2016.
23. Platt D, Amara S, Mehta T, Vercuyssee K, Myles EL, Johnson T and Tiriveedhi V: Violacein inhibits matrix metalloproteinase mediated CXCR4 expression: Potential anti-tumor effect in cancer invasion and metastasis. Biochem Biophys Res Commun 455: 107-112, 2014.

24. Xu C, Zhao H, Chen $\mathrm{H}$ and Yao Q: CXCR4 in breast cancer: Oncogenic role and therapeutic targeting. Drug Des Devel Ther 9: 4953-4964, 2015.

25. Szallasi Z, Krsmanovic L and Blumberg PM: Nonpromoting 12-deoxyphorbol 13-esters inhibit phorbol 12-myristate 13 -acetate induced tumor promotion in CD-1 mouse skin. Cancer Res 53: 2507-2512, 1993.

26. Wang MT, Holderfield M, Galeas J, Delrosario R, To MD, Balmain A and McCormick F: K-Ras Promotes Tumorigenicity through Suppression of Non-canonical Wnt Signaling. Cell 163: 1237-1251, 2015.

27. Biancotto A, Grivel JC, Gondois-Rey F, Bettendroffer L, Vigne R, Brown S, Margolis LB and Hirsch I: Dual role of prostratin in inhibition of infection and reactivation of human immunodeficiency virus from latency in primary blood lymphocytes and lymphoid tissue. J Virol 78: 10507-10515, 2004.

28. Hiller D and Chu QD: CXCR4 and axillary lymph nodes: Review of a potential biomarker for breast cancer metastasis. Int J Breast Cancer 2011: 420981, 2011. 